\title{
On the far-infrared metallicity diagnostics: applications to high-redshift galaxies
}

\author{
D. Rigopoulou, ${ }^{1 \star}$ M. Pereira-Santaella ${ }^{1}$, G.E. Magdis ${ }^{2}$, A. Cooray ${ }^{3}$, D. Farrah ${ }^{4}$, \\ R. Marques-Chaves ${ }^{5,6}$, I. Perez-Fournon ${ }^{5,6}$, D. Riechers ${ }^{7}$ \\ ${ }^{1}$ Astrophysics, Department of Physics, University of Oxford, Keble Road, Oxford, OX1 3RH, UK \\ ${ }^{2}$ Dark Cosmology Centre, Niels Bohr Institute, University of Copenhagen, Juliane Mariesvej 30, DK-2100 Copenhagen, Denmark \\ ${ }^{3}$ Department of Physics \& Astronomy, University of California, Irvine, CA 92697, USA \\ ${ }^{4}$ Department of Physics, Virginia Tech, Blacksburg, VA 24061, USA \\ ${ }^{5}$ Instituto de Astrofisica de Canarias, E-38205 La Laguna, Tenerife, Spain \\ ${ }^{6}$ Universidad de La Laguna, Dpto. Astrofisica, E-38206 La Laguna, Tenerife, Spain \\ ${ }^{7}$ Department of Astronomy, Cornell University, 220 Space Sciences Building, Ithaca, NY 14853, USA
}

Accepted XXX. Received YYY; in original form ZZZ

\begin{abstract}
In an earlier paper we modeled the far-infrared emission from a star-forming galaxy using the photoionization code CLOUDY and presented metallicity sensitive diagnostics based on far-infrared fine structure line ratios. Here, we focus on the applicability of the $[\mathrm{OIII}] 88 \mu \mathrm{m} /[\mathrm{NII}] 122 \mu \mathrm{m}$ line ratio as a gas phase metallicity indicator in high redshift submillimetre luminous galaxies. The [OIII] $88 \mu \mathrm{m} /[\mathrm{NII}] 122 \mu \mathrm{m}$ ratio is strongly dependent on the ionization parameter (which is related to the total number of ionizing photons) as well as the gas electron density. We demonstrate how the ratio of $88 \mu \mathrm{m} / 122 \mu \mathrm{m}$ continuum flux measurements can provide a reasonable estimate of the ionization parameter while the availability of the [NII]205 $\mu \mathrm{m}$ line can constrain the electron density. Using the $[\mathrm{OIII}] 88 \mu \mathrm{m} /[\mathrm{NII}] 122 \mu \mathrm{m}$ line ratios from a sample of nearby normal and star-forming galaxies we measure their gas phase metallicities and find that their mass metallicity relation is consistent with the one derived using optical emission lines. Using new, previously unpublished, Herschel spectroscopic observations of key far-infrared fine structure lines of the $\mathrm{z} \sim 3$ galaxy HLSW-01 and additional published measurements of far-infrared fine structure lines of high-z submillimetre luminous galaxies we derive gas phase metallicities using their [OIII] 88 $\mu \mathrm{m} /[\mathrm{NII}] 122 \mu \mathrm{m}$ line ratio. We find that the metallicites of these $\mathrm{z} \sim 3$ submm luminous galaxies are consistent with solar metallicities and that they appear to follow the mass-metallicity relation expected for $\mathrm{z} \sim 3$ systems.
\end{abstract}

Key words: galaxies: high-redshift - galaxies: ISM - galaxies: abundances - infrared: galaxies - submillimetre: galaxies - ISM: lines and bands

\section{INTRODUCTION}

The metallicity of a galaxy is closely linked to its star formation history and the inflow and outflow of gas, the interplay between the galaxy's insterstellar medium (ISM) and the intergalactic medium (IGM). Gas accretion from the IGM to the ISM together with large scale outflows are the two principal components in galaxy formation and evolution models (e.g. Dekel et al. 2009, Davé et al. 2012). Feedback from star formation drives outflows that remove mass and metals away from galaxies, whereas, infall of gas from the IGM to

^ E-mail:dimitra.rigopoulou@physics.ox.ac.uk the ISM is necessary to sustain star-formation. Without the feedback, baryons would cool into the centers of haloes and form prodigious amounts of stars (e.g. Keres et al. 2009) but with feedback the baryonic content of stars and cold gas in galaxies can be reconciled by driving matter into the IGM (e.g. Conroy \& Wechsler 2009). Likewise, without infall of material from the IGM star forming galaxies would use up their ISM gas in $~ 1$ Gyr (e.g. Genzel et al. 2010). To understand these processes we need to study how galaxies obtain, process, expel and recycle gas from their surroundings.

Galaxies evolve through the build-up of stellar mass $\left(\mathrm{M}_{*}\right)$ that takes place over time through a number of star formation episodes. As stars evolve they produce metals so 
that the metal content of a galaxy, typically measured by the oxygen abundance $(\mathrm{O} / \mathrm{H})$, can be used as a diagnostic for tracing galaxy formation and evolution. The presence of a tight correlation between the stellar mass of a galaxy and its gas-phase metallicity $(\mathrm{Z})$, the so called $\mathrm{M}_{*}-\mathrm{Z}$ relation has been found to hold in the nearby Universe (Tremonti et al. 2004) and up to redshifts $\mathrm{z} \sim 2$ (e.g. Erb et al. 2006, Maiolino et al. 2008, Wuyts et al. 2014) and z 3 (e.g. Mannucci et al 2009, Onodera et al. 2016). Mannucci et al. (2010) extended this correlation to include the star formation as well, defining the so-called fundamental metallicity relation (FMR).

Gas phase galaxy metallicities have traditionally been derived using ratios between strong optical emission lines ([OII] $] \lambda 3727 \dot{\mathrm{A}},[\mathrm{OIII}] \lambda 5007 \dot{\mathrm{A}}, \mathrm{H}_{\alpha}, \mathrm{H}_{\beta}$, [NII] $\left.\lambda \lambda 6549 \dot{\mathrm{A}}, 6583 \dot{\mathrm{A}}\right)$ calibrated on theoretical models (e.g., Kewley \& Dopita 2002; Kewley \& Ellison 2008; Maiolino et al. 2008). The most commonly used ones are those based on the "strong line methods" and include the R23 diagnostics (combining [OIII], [OII], and $\mathrm{H}_{\beta}$; Pagel et al. 1979) and the N2 method (combining $\mathrm{H}_{\alpha}$ and [N II]; Storchi-Bergmann et al. 1994). The N2 method is commonly used to break the degeneracy and dust dependency of the R23 method. The strong-line methods can be used for galaxies up to $\mathrm{z} \sim 3$, at higher redshifts however, the diagnostic lines shift out of the wavelengths accessible by ground-based near- IR spectrographs. A correlation between metallicity and the equivalent width (EW) of absorption features in the rest-frame ultraviolet (UV) is expected based on theoretical models (e.g. Eldridge \& Stanway 2012) and is observed in local starburst galaxies (e.g. Heckman et al. 1998, Leitherer et al. 2011). This method provides a way to probe statistically the metal content of galaxies and has been used in $\mathrm{z} \sim 3$ galaxies (e.g. Savaglio et al. 2004, Sommariva et al. 2012) and more recently by Faisst et al. (2016) for $\mathrm{z} \sim 5$ galaxies, however, its use remains limited due to the faintness of the features. As a result little is known of the metal content of galaxies at very early epochs and therefore our understanding of the formation of these galaxies is incomplete.

An alternative way to derive gas phase metallicities relies on the use of far-infrared (FIR) atomic fine structure (FS) line transitions, [OIII] 52 and $88 \mu \mathrm{m}$, [NIII] $57 \mu \mathrm{m}$, [NII]122 and $205 \mu \mathrm{m}$, which are less susceptible to extinction compared to optical and UV lines. These lines originate primarily in [HII] regions, their emission is well understood and can be easily modeled. For nearby galaxies these lines are only accessible from space or stratospheric platforms such as SOFIA (Gehrz et al., 2009). The recent advent of the Herschel mission (Pilbratt et al. 2010) and before that, of ISO (Kessler et al. 1996) provided measurements of these key FIR FS lines in several nearby galaxies. Herschel was also able to detect FIR FS lines in a handful of gravitationally lensed sources at redshifts between $2<z<3$. For distant galaxies, and, especially those at redshifts $\mathrm{z}>4$ where optical line metallicity diagnostics are not available, the FIR FS lines shift into the submillimetre range and are now accessible with the Atacama Large Millimetre Array (ALMA) and the Northern Extended Millimetre Array (NOEMA).

Pereira Santaella et al. 2017 (hereafter PS17) used the photoionization code CLOUDY (Ferland et al. 2013) to model the FIR FS emission lines produced in HII regions as a function of the metallicity, density, and ionization parameter. The lines considered were: [OIII] 52 and $88 \mu \mathrm{m}$,
[NIII] $57 \mu \mathrm{m}$ and [NII] 122 and $205 \mu \mathrm{m}$. A direct comparison of the observed fine structure lines ratios to model predictions allow the gas phase metallicity to be determined once a value for the density and the ionization parameter has been established. PS17 used FIR FS metallicity ratios to succesfully determine gas phase metallicities in a sample of local ultraluminous infrared galaxies (ULIRGs). The technique outlined in PS17 can be used to estimate the metal content of heavily dust-obscured galaxies, like the submmluminous galaxies, where tranditional methods relying on optical or UV line diagnostics may fail.

Wide area submillimetre surveys carried out with Herschel, such as the Herschel Astrophysical Terahertz Large Area Survey (H-ATLAS; Eales et al. 2010) and the Herschel Multi-Tiered Extragalactic Survey (HerMES; Oliver et al. 2012), have resulted in the dicovery of large numbers of dusty star-forming galaxies. HLSW-01 is the brightest gravitationally lensed source discovered by HerMES. With a magnification of $\mu=10.9 \pm 0.7$ (Gavazzi et al. 2011), HLSW01 has been the subject of significant follow-up effort. SMA interferometric observations (Conley et al. 2011) resolved the source in four components while Keck-II/NIRC2 K-band imaging resolved the structures further. The NIRC2 data showed that the galaxy is magnified by a small group of $\mathrm{z} \sim 0.6$ galaxies. Based on IFU observations of the brightest foreground galaxy with Oxford-SWIFT (Thatte et al. 2006) we measured a spectroscopic redshift of 0.64 for the brightest member of the group (Foster et al. 2017, in prep). The redshift of HLSW-01 was established from $\mathrm{CO}$ emission lines as $\mathrm{z}=2.958$ (Scott et al. 2011, Riechers et al. 2011). A detailed analysis of the dynamics of the gas showed that the system is consistent with a gas rich merger at $\mathrm{z} \sim 3$. More recent spectroscopic observations with the GTC have confirmed the merging nature of HLSW-01 (Marques-Chaves et al. 2017, in prep).

In this paper we examine the applicability of the $[\mathrm{OIII}] 88 \mu \mathrm{m} /[\mathrm{NII}] 122 \mu \mathrm{m}$ line ratio as a metallicity indicator in galaxies near and far. We use the aforementioned line ratio to calibrate and derive a metallicity diagnostic for local normal and star-forming galaxies and then extend the method to distant galaxies. Using previously unpublished Herschel measurments of FIR FS lines from HLSW-01 and published data from distant dusty submm-luminous galaxies we determine, for the first time, their metallicity and examine their location on the MZR plane. The paper is organised as follows: new Herschel measurements of the FIR FS lines in HLSW-01 are presented in Section 2. In Section 3 we summarise the CLOUDY models and present a new method to estimate the ionization parameter, the gas electron density and, determine gas phase metallicities. In Section 4 we apply our method to a sample of nearby galaxies and to a number of distant submm-luminous galaxies. In Section 5 we summarize the main findings of this work. Throughout this paper, we adopt a cosmology with $\mathrm{H}_{0}=70 \mathrm{~km} \mathrm{~s}^{-1} \mathrm{Mpc}^{-1}$, $\Omega_{\Lambda}=0.7$, and $\Omega_{m}=0.3$.

\section{SPIRE-FTS OBSERVATIONS OF HLSW-01}

Observations of HLSW-01 were taken on Ferbuary 20th 2013, as part of our Herschel (Pilbratt et al. 2010) OT2 program on far-infrared spectroscopic observations of ultra- 
luminous infrared galaxies (Rigopoulou et al. 2014). HLSW01 was observed for $4 \mathrm{hrs}$ with a single pointing of the SPIRE Fourier Transform Spectrometer (FTS, Griffin et al. 2010) sampling across a field of view of $2.6^{\prime}$ in diameter. The instrument was used in the high spectral resolution mode. The SPIRE-FTS employs two detector arrays covering the 194$313 \mu \mathrm{m}$ (SSW) and 303-671 $\mu \mathrm{m}$ (SLW) wavelength bands simultaneously to measure the Fourier transform of the spectrum of a source. The FWHM beamwidths of the SSW and SLW arrays vary from 17 " at $194 \mu \mathrm{m}$ to 42 " at $671 \mu \mathrm{m}$ in a complex fashion due to the nature of the SPIRE detectors (Swinyard et al. 2014). The data were processed with the SPIRE pipeline (Foulton et al. 2014) in HIPE (Ott et al. 2010) version 12.1. Since the majority of the targets in our sample are considered 'faint' targets for the FTS post pipeline processing beyond the standard reduction has been necessary. A detailed account of the steps followed to reduce the spectra can be found in Magdis et al. (2014). We examined the reduced spectra for spectral features at the positions of expected lines. To increase the reliability of the line detections and/or minimise the chance of spurious detections we applied the Jackknife tecnhique (described in detail in Magdis et al. 2014). Using this method, decreasing number of scans (down to ten) are averaged and examined for reccuring peaks. A real line shows as a consistent peak at the expected position in all the subsets examined. To measure reliable line fluxes and background levels bootstrapping was used. For each observation the unaveraged scans were randomly sampled until we reached the number in the parent population (of 200). These scans were then averaged and line measurements taken using the same basic fitting technique as for a standard average spectrum. Baseline subtraction was performed to each scan prior to resampling. The mean line flux was obtained through resampling of each observation and then Gaussian fitting to the resulting line flux. The Gaussian width was set at the associated $1 \sigma$ uncertainties. The background level above which spectral features were detected was established from the distributions obtained from the set of random frequency positions. The instrumental line shape of the FTS is well approximated by a sinc function (see Swinyard et al.2014 and Hopwood et al. 2014 for more details). For unresolved lines, a sinc function was fitted simultaneously with a $3^{\text {rd }}$ order polynomial for the continuum. If the line was partially resolved then we employed a sinc convolved with a Gaussian using the SINC-GAUSS function within HIPE. In total we detect the $[\mathrm{OIII}] 51.81 \mu \mathrm{m},[\mathrm{NIII}] 57.8 \mu \mathrm{m},[\mathrm{OI}] 63 \mu \mathrm{m},[\mathrm{OIII}] 88 \mu \mathrm{m}$ and, [CII] $158 \mu \mathrm{m}$ lines at $>3 \sigma$ while for [NII] $122 \mu \mathrm{m}$ and [OI] $145 \mu \mathrm{m}$ lines we report a $3 \sigma$ upper limit. In Table 1 we provide the lines fluxes measured at the expected frquencies. All lines have been detected at the expected frequency, corresponding to the redshift of the source of $\mathrm{z}=2.9574$, except for the $[\mathrm{OI}] 63 \mu \mathrm{m}$ line which was detected at a frequency corresponding to a redshift of $\mathrm{z}=2.9513$. In Figure 1 we show the full SPIRE-FTS spectrum while in Figure 2 we plot the continuum substracted emission line profiles together with the best fit models.

The FIR FS lines provide useful insight into the properties of the ISM and the underlying ionizing source in HLSW01. Conley et al. (2011) \& Magdis et al. (2014) reported on the unusually warm colours of HLSW-01 which would imply the presence of an AGN. The [OI] $63 \mu \mathrm{m} /$ [CII] 158
Table 1. Line measurements and upper limits for the FIR FS lines detected in HLSW-01

\begin{tabular}{lcccc}
\hline $\begin{array}{l}\text { Line ID } \\
\mu \mathrm{m}\end{array}$ & $\begin{array}{c}\text { Obs. Freq. } \\
\mathrm{GHz}\end{array}$ & $\begin{array}{c}\text { Flux } \\
\times 10^{-18} \mathrm{~W} \mathrm{~m}^{-2}\end{array}$ & $\begin{array}{c}\text { FWHM } \\
\mathrm{km} \mathrm{s}^{-1}\end{array}$ & SNR \\
\hline & & & & \\
{$[\mathrm{O}$ III $] 51.88$} & 1462.60 & $6.75 \pm 0.78$ & 293.06 & 8.0 \\
{$[\mathrm{~N}$ III $] 57.3$} & 1329.52 & $4.42 \pm 1.23$ & 364.03 & 3.46 \\
{$[\mathrm{O}$ I] 63.2} & 1198.95 & $8.43 \pm 0.78$ & 357.32 & 5.88 \\
{$[\mathrm{O}$ III $] 88$} & 857.31 & $8.38 \pm 1.04$ & 499.83 & 8.0 \\
{$[\mathrm{C}$ II $] 157.7$} & 480.44 & $9.06 \pm 2.79$ & 896.0 & 3.72 \\
\hline
\end{tabular}

\begin{tabular}{lcccc} 
Upper Limits & & & & \\
\hline$[\mathrm{N}$ II $] 122.1$ & 618.45 & $<4.08$ & 425.0 & 3.0 \\
{$[\mathrm{O}$ I 145.5} & 520.61 & $<2.098$ & 896.0 & 3.0 \\
\hline
\end{tabular}

$\mu \mathrm{m}$ ratio can be used to characterise the nature of the underlying radiation field (e.g. Abel et al. 2009). Based on the values reported in Table 1 we calculate a ratio of 0.93 which is more typical of excitation by a starburst rather than an AGN. On the other hand, the [OIII] $88 \mu \mathrm{m} / \mathrm{FIR}$ ratio is $1.56 \times 10^{-3}$. Brauher et al. (2008) found high values of the $[\mathrm{OIII}] 88 \mu \mathrm{m} / \mathrm{FIR}$ ratio in galaxies with warm $60 \mu \mathrm{m} / 100$ $\mu \mathrm{m}$ colours, attributing this increase either to a higher density of HII regions or the presence of an AGN. Hence, while HLSW-01 may contain an AGN it is unlikely that the AGN is contributing significantly to the FIR FS lines.

\section{FAR-IR FINE STRUCTURE EMISSION LINE METALLICITY DIAGNOSTICS}

\subsection{CLOUDY models and metallicity sensitive FIR FS line ratios}

In Pereira-Santaella et al. (2017, hereafter PS17) we used the spectral synthesis photoionization code CLOUDY (Ferland et al. 2013) to model the far-IR fine structure emission lines produced in HII regions. We examined how combinations of these far-IR fine structure lines can be used to constrain gas phase metallicities.

In brief, CLOUDY computes the chemical and thermal structure of a cloud from the illuminated face to regions of high column density deep into the clouds where atoms combine to form molecules. CLOUDY incorprorates photoelecting heating of the gas by grains, cosmic ray ionization and heating, in addition to photoionization and photodissociation processes. Details of the processes can be found in van Hoof et al. (2004), Abel et al. (2005) and in Ferland et al. (2013). In PS17 we considered constant pressure models illuminated by a continuous burst of star formation. The input spectrum has been calculated using STARBURST99, Leitherer et al. (1999). Gas phase abundances were matched to those of the incident spectrum. For solar metallicity, the values reported in Asplund et al. (2009) were used while, for the remaining metallicities we assumed that abundances scale as $\mathrm{Z}_{\text {gas }} / \mathrm{Z}_{\odot}$ for all the elements except for He and $\mathrm{N}$ (values for the latter come from Dopita et al. 2006). Dust is also included in the models following the prescription of Remy- 


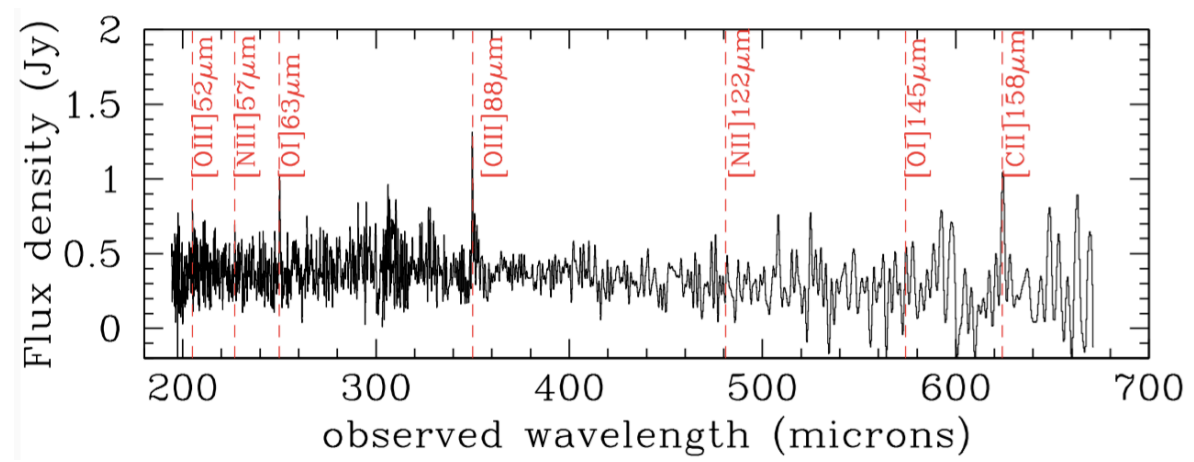

Figure 1. The full SPIRE-FTS spectrum of HLSW-01 in the observed frame. The vertical lines correspond the wavelengths of [OIII] 52 , [NIII] $57,[\mathrm{OI}] 63,[\mathrm{OIII}] 88,[\mathrm{NII}] 122,[\mathrm{OI}] 145$ and [CII]158 microns.
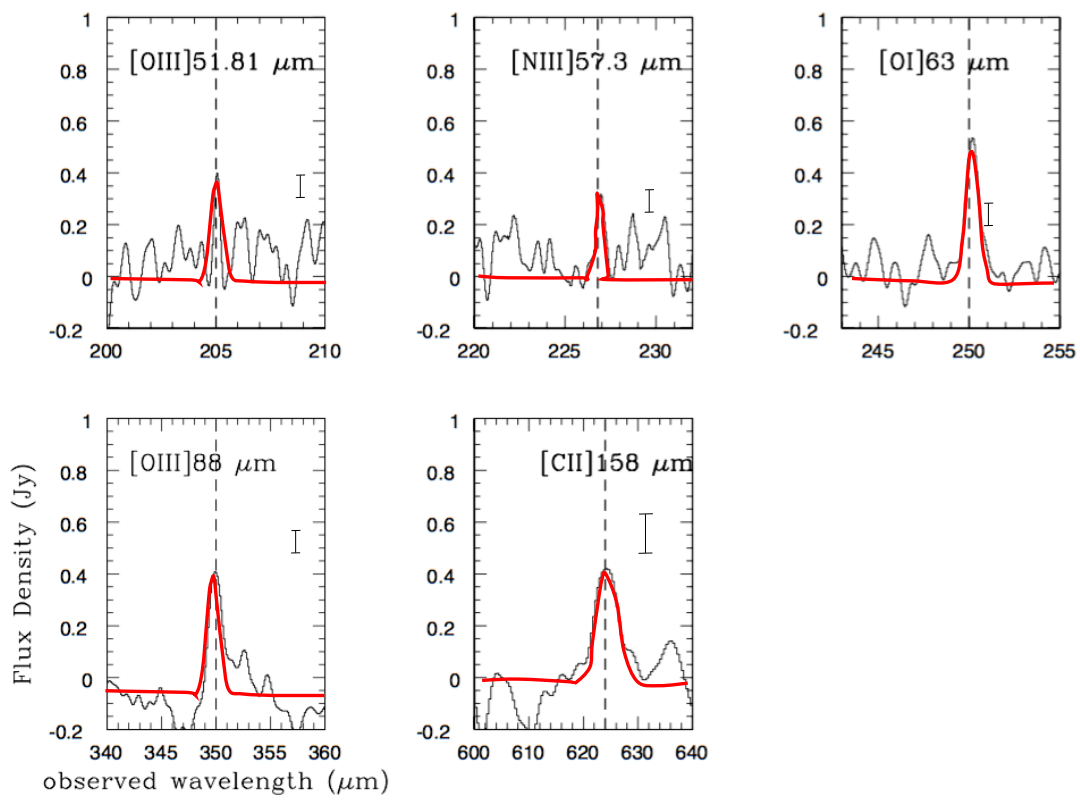

Figure 2. Continuum-subtracted SPIRE-FTS spectra of HLSW-01 around the expected wavelengths for (clockwise from top) [OIII]51.81 $\mu \mathrm{m},[\mathrm{NIII}] 57.3 \mu \mathrm{m},[\mathrm{OI}] 63.2 \mu \mathrm{m},[\mathrm{OIII}] 88 \mu \mathrm{m}$ and [CII] $157.7 \mu \mathrm{m}$. The dashed line indicated the expected wavelength of each line.

Ruyer et al. (2014). A detailed description of the model assumptions can be found in PS17.

The three most important parameters of the CLOUDY models are the gas volume density $\mathrm{n}_{\mathrm{H}}$ (in units of $\mathrm{cm}^{-3}$, hereafter gas density), the ionization parameter $\mathrm{U}$ (defined as the ratio of hydrogen-ionizing photon density to gas density, $\left.\mathrm{U}=\phi /\left(\mathrm{n}_{\mathrm{H}} \mathrm{c}\right)\right)$ and, the gas phase metallicities. Constraining the model values for $\mathrm{U}$ and $\mathrm{n}_{\mathrm{H}}$ allows us to determine gas phase metallicities through comparison of the observed line ratios to model predictions.

Amongst the various combinations of FIR FS line ratios considered we found that, the [OIII] to [NIII] ratios are the best metallicity tracers. This is because the $\mathrm{O}^{++}$and $\mathrm{N}^{++}$ lines that dominate the emission in HII regions have very similar ionization potentials: $35.12 \mathrm{eV}$ and $29.6 \mathrm{eV}$ for the [OIII] 52 and $88 \mu \mathrm{m}$ and [NIII] $57 \mu \mathrm{m}$, respectively. Because the ionization potentials are rather similar, their ratio is independent of the specific model value of the ionization parameter although it shows a mild dependency on the model value of the gas density. However, the density dependence of the $[\mathrm{OII}] 52 \mu \mathrm{m} /[\mathrm{NIII}] 57 \mu \mathrm{m}$ ratio is opposite to that of the [OIII] $88 \mu \mathrm{m} /[\mathrm{NIII}] 57 \mu \mathrm{m}$ ratio because the [OIII] $52 \mu \mathrm{m}(88$ $\mu \mathrm{m}$ ) is enhanced at high (low) densities. As demonstrated in PS17, the formula $(2.2 \times[\mathrm{OIII}] 88+[\mathrm{OIII}] 52) /[\mathrm{NII}] 57$ combining all three lines, reduces the scatter produced by the dependency on the densities. In section 4.2 we use this ratio to determine the metallicity of HLSW-01 using the Herschel FIR FS lines presented here.

\subsection{The $[\mathrm{OIII}] 88$ to $[\mathrm{NII}] 122$ line ratio}

The [OIII] 52 and [NIII] $57 \mu \mathrm{m}$ lines are not easily accessible from the ground; only for galaxies at $\mathrm{z}>5$ do the lines shift into windows accessible to ALMA. Here, we consider the [OIII] $88 /[\mathrm{NII}] 122$ line ratio as a potential gas phase metallicity diagnostic ratio particularly suitable for high redshift galaxies where both lines shift into the wavelength coverage of ALMA. The [OIII] $88 \mu \mathrm{m}$ and $[\mathrm{NII}] 122 \mu \mathrm{m}$ lines have 
similar critical densities, around $300-500 \mathrm{~cm}^{-3}$, hence, the [OIII]/[NII] ratio does not depend strongly on the model gas density value. However, the ratio has a strong dependency on the ionization parameter $U$ since the number of ionizing photons available will stronly influence the relative amounts of $\mathrm{O}^{++}$and $\mathrm{N}^{+}$gas present. Therefore, in order to use this metallicity diagnostic ratio we need to determine the value of the ionization parameter U. In what follows we present a method that allows us to establish the value of $U$ based on available continuum measurements.

\subsubsection{The ionization parameter}

Combinations of mid and far-infrared transitions have been found to correlate with the ionization parameter U. For instance, the $[\mathrm{NeII}] 12.8 \mu \mathrm{m} /[\mathrm{NeIII}] 15.5 \mu \mathrm{m}$ ratio (e.g. Rigby \& Rieke 2004) or the [SIV]10.5 $\mu \mathrm{m} /[\mathrm{NeIII}] 15.5 \mu \mathrm{m}$ ratio (PS17) have been found to provide good constraints on the value of the ionization parameter. However, most of these lines are not accessible with current space or ground-based facilities. The lines will become within the reach of JWST but only for $z<2$ galaxies.

The 60 -to-100 $\mu \mathrm{m}$ flux density ratio (commonly referred to as $\mathrm{C}(60 / 100)$ or colour index) has long been established as a tracer of the dust temperature $\mathrm{T}_{\text {dust }}$ of a galaxy (e.g. Chanial et al. 2007). Furtheromore, it has been shown (e.g. Abel et al. 2009, Fischer et al. 2014) that, C(60/100) varies strongly with the ionization parameter $\mathrm{U}$ and, therefore, can be used to constrain its value. As U increases, C(60/100) increases as well, because the total flux of ionizing photons goes up. An increase in the total ionizing flux will provide additional heating to the gas and dust and, therefore, increase the dust temperature. Since the dust temperature increases the dust emits more energy at shorter wavelengths and the flux at $60 \mu \mathrm{m}$ will be higher relative to the flux at $100 \mu \mathrm{m}$.

But although the detailed Spectral Energy Distribution (SED) and the $\mathrm{C}(60 / 100)$ are usually widely available for local galaxies this is not the case for many distant galaxies where only measurements of FIR FS lines may exist with little or no information on the shape of the SED of the galaxy. In this case, it is instructive to investigate whether we can use the 88 -to- $122 \mu \mathrm{m}$ contonuum flux density ratio (hereafter $\mathrm{C}(88 / 122))$ to determine the value of the ionization parameter. To assess whether the $\mathrm{C}(88 / 122)$ is a viable alternative to $\mathrm{C}(60 / 100)$ we first compare the dust temperatures derived from fitting 60 and $100 \mu \mathrm{m}$ and, 88 and $122 \mu \mathrm{m}$ continuum flux densities in each case with a modified black body (MBB) with a fixed dust emissivity index of $\beta=1.5$. We use the sample of nearby normal and starburst galaxies from Brauher et al. (2008) where both sets of measurements, 60 and $100 \mu \mathrm{m}$ from IRAS and, 88 and $122 \mu \mathrm{m}$ continuum measurements (derived from ISO-LWS spectra) are available. For our investigation we select only galaxies that are unresolved within the ISO-LWS beam and where measurements of the continuum at 88 and $122 \mu \mathrm{m}$ are available. It is important for the present study that the galaxies considered are unresolved within the ISO beam so that line emission from the entire galaxy is considered. This has the obvious disadvantage that we cannot explore metallicity gradients however, FIR FS lines are usually not subject to extinction therefore, the lines could be tracing the emission from all

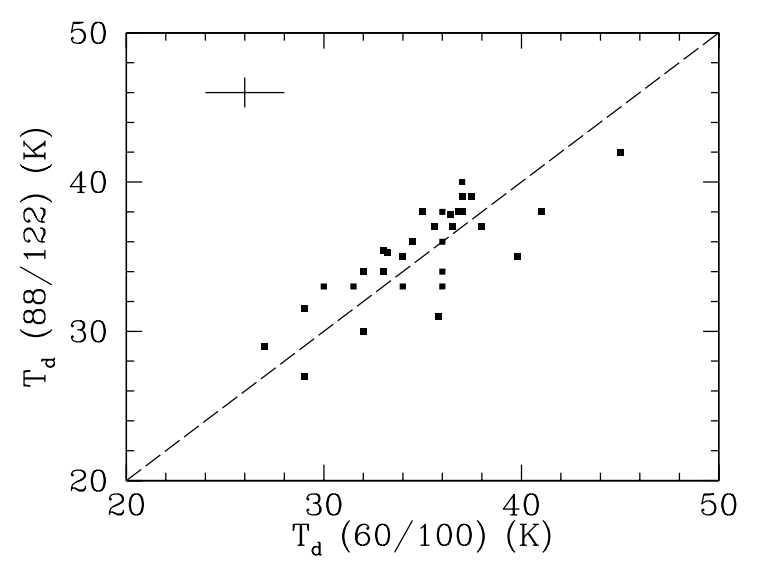

Figure 3. Temperature of the modified blackbodies $(\beta=1.5)$ fitted to the 60 and $100 \mu \mathrm{m}$ flux densities (abscissae) and to the 88 and 122 continuum fluxes (ordinates) for the sample of local normal and star-forming galaxies with ISO measurements (from Brauher et al. 2008). The dashed line is the unity line and the average uncertainties are shown in the upper left corner.

parts of the galaxy irrespective of extinction. An exception are sources like Arp 220 where the dust optical depth reaches unity around $200 \mu \mathrm{m}$ and therefore, extinction could become an issue (e.g. Riechers et al. 2013). In total there are 40 such galaxies where continuum measurements at 88 and $122 \mu \mathrm{m}$ are available. The galaxies considered here are all local, the highest redshift is $\mathrm{z}=0.0143$. The $\mathrm{C}(60 / 100)$ for these galaxies ranges between $0.32-1.37$ while the $\mathrm{C}(88 / 122)$ is in the range of $0.67-1.97$. As mentioned above, we assume a modified blackbody (MBB) and a dust emissivity of $\beta=1.5$ to convert the measured $\mathrm{C}(60 / 100)$ and $\mathrm{C}(88 / 122)$ flux ratios to dust temperatures $\mathrm{T}_{d}$, respectively. In Figure 3 we plot the temperatures of the MBB fitted to the 60 and $100 \mu \mathrm{m}$ fluxes (abscissae) and 88 and $122 \mu$ m fluxes (ordinates). The standard deviation is $2.8 \mathrm{~K}$ which is small enough to validate the use of $\mathrm{C}(88 / 122)$ as a proxy for $\mathrm{C}(60 / 100)$ and therefore an estimator of the dust temperature of galaxies.

To further explore how $\mathrm{C}(88 / 122)$ varies as a function of the ionization parameter U, we use the CLOUDY models outlined in section 3.1 to compute the variation of the $\mathrm{C}(88 / 122)$ index as a function of the $\mathrm{U}$ parameter. In Figure 4 we plot model values for the $\mathrm{C}(88 / 122)$ as a function of the ionization parameter $\mathrm{U}$ for three different values of the gas density. We confirm the trend that the $\mathrm{C}(88 / 122)$ increases with increasing values of $U$ however, we also note that the slope becomes shallower for lower values of the gas density. The models shown in Figure 4 assume an $A_{V} \sim 100$. This value of $A_{V}$ was chosen since it is known that extinction toward the most obscured star-forming regions in galaxies can reach up to such high values (e.g. Abel et al. 2009). A higher (lower) value of $\mathrm{A}_{V}$ will make the $\mathrm{C}(88 / 122)$ ratio grow slower (faster) as a function of increasing ionization parameter U.

\subsubsection{The gas density}

The value of the gas density, $\mathrm{n}_{\mathrm{H}}$, can be determined through ratios of mid and/or far-IR fine structure lines from the same 


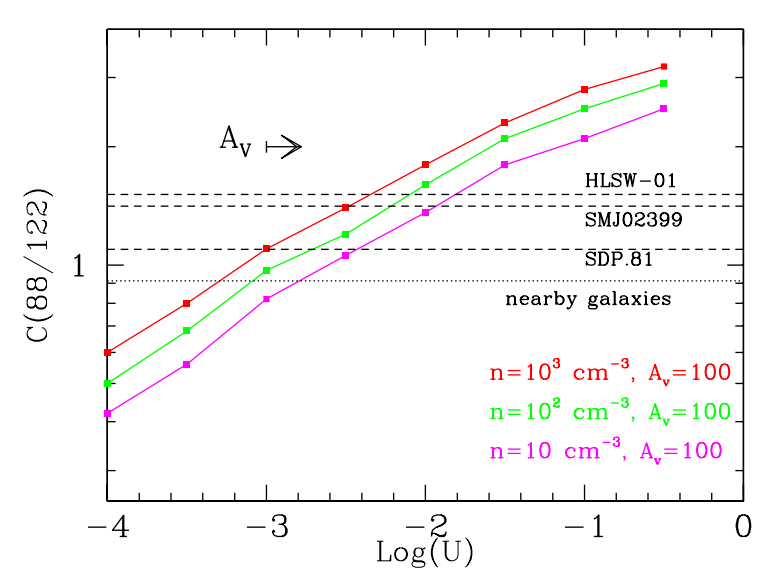

Figure 4. Model predicted $\mathrm{C}(88 / 122)$ flux ratio as a function of the logarithm of the ionization parameter, $\log (\mathrm{U})$. The plot shows three tracks each for different gas density of $10,100,1000 \mathrm{~cm}^{-3}$ and $\mathrm{A}_{V}=100$. The dashed lines indicate the $\mathrm{C}(88 / 122)$ for the three high-z submm galaxies while the dotted line indicated the median value for the ISO sample of nearby galaxies. The effect of an increasing $A_{V}$ value is indicated with the arrow.

ion but with different critical densities. If both [NII] lines 122 and $205 \mu \mathrm{m}$ are available then their ratio provides a good estimate of the gas density especially at the low density regime (see Oberst et al. 2006). Alternatively, if no other FIR FS lines are available a value of $\sim 100 \mathrm{~cm}^{-3}$ can be assumed, as has been found by many independent studies of determining gas densities in other galaxy samples (Section 4.1).

In summary, we have shown that if the [OIII] $88 /[\mathrm{NII}] 122$ line ratio is used to determine gas phase metallicities then the ratio of the continuum fluxes $\mathrm{C}(88 / 122)$ can be used to constrain the value of the ionization parameter $\mathrm{U}$. This is particularly useful for high-z sources where detailed information on the shape of the SED of the source may not be available.

\section{RESULTS}

\subsection{IR Metallicities of nearby normal and starburst galaxies}

In this section we apply the diagnostics described above to derive gas phase metallicities of a sample of local normal and star-forming galaxies and individual high-z submm galaxies with available measurements of the FIR FS lines. Brauher et al. (2008) reported measurements of the [OIII] 88 $\mu \mathrm{m}$ and $[\mathrm{NII}] 122 \mu \mathrm{m}$ emission lines for 30 nearby normal and starburst galaxies (we chose those galaxies where both lines are detected at $\mathrm{S} / \mathrm{N}>3$ ). Following the discussion in Section 3 to determine gas phase metallicities through comparison of the observed [OIII] $88 /[\mathrm{NII}] 122$ line ratio to CLOUDY model predictions, the gas density and the ionization parameter need to be established. The Brauher et al. paper does not report measurements of the $[\mathrm{NII}] 205 \mu \mathrm{m}$ lines. Instead we searched the Herschel archive for measurements of the $[\mathrm{NII}] 205 \mu \mathrm{m}$ line. Only 10 of the galaxies with values of the [OIII] 88/[NII]122 reported in Brauher et al. have [NII]205 $\mu \mathrm{m}$ values measured by Herschel. In deriving the
[NII]122/205 ratio we applied corrections to account for the difference in the size of beams (between ISO and Herschel) following the procedure described in Rigopoulou et al. (2013). We measure [NII] 122/205 line ratios of 0.6-4 with a median ratio of $\sim 1.8$ corresponding to an $n_{H}$ value of $\sim$ $60 \mathrm{~cm}^{-3}$. A value of $1-300 \mathrm{~cm}^{-3}$ with a median value of $30 \mathrm{~cm}^{-3}$ was derived by Herrera-Camus et al. (2016) for the KINGFISH sample of nearby galaxies. PS17 adopted a median value of $n_{H} \sim 100 \mathrm{~cm}^{-3}$, for a sample of ultraluminous infrared galaxies, derived from the $[\mathrm{NII}] 122 /[\mathrm{NII}] 205 \mu \mathrm{m}$ ratio which is a good probe of the gas density especially at the low density limit (e.g. Oberst et al. 2006). We conclude that a value of $\mathrm{n}_{H} \sim 100 \mathrm{~cm}^{-3}$ is a good representative value for the gas density of normal and starburst galaxies.

Turning to the ionization parameter, in Section 3 we discussed how the continuum ratio $\mathrm{C}(88 / 122)$ can be used to constrain the value of the ionization parameter. The median $\mathrm{C}(88 / 122)$ value for the Brauher et al. sample of galaxies is 0.908 (ranging between 0.72 and 1.46) which, for a gas density of $100 \mathrm{~cm}^{-3}$, corresponds to the logarithm of the ionization parameter, $\log (\mathrm{U})$, between -3.6 to -2.2 .

Figure 5 shows model predictions for the [OIII] 88 $\mu \mathrm{m} /[\mathrm{NII}] 122 \mu \mathrm{m}$ line ratio as a function of metallicity for various values of the ionization parameter $U$ (the compilation of the model values used can be found in Table A1 of the Appendix of PS17). In the Brauher et al. sample of nearby normal and starburst galaxies the [OIII] $88 /[\mathrm{NII}] 122$ line ratio varies between 1.15 - 10.63 with a median value of 2.73. Assuming $\log (\mathrm{U})$ values in the range $-3.6<\log (\mathrm{U})<-2.2$ the median $[\mathrm{OIII}] /[\mathrm{NII}]$ line ratio of 2.73 implies metallicities in the range $0.6<\mathrm{Z}_{\text {gas }} / Z_{\odot}<1.0$ which correspond to oxygen abundances of $8.46<\log (\mathrm{O} / \mathrm{H})<8.69$. These metallicity estimates are consistent with those based on optical diagnostics presented for some of these galaxies in Moustakas \& Kennicutt (2006).

\subsection{IR metallicities of dusty high-z galaxies}

Metallicity estimates for large samples of $0.5<\mathrm{z}<2$ galaxies have been derived from $\mathrm{R}_{23}$ or from $[\mathrm{NII}] 6584 / \mathrm{H}_{\alpha}$ ratio (e.g. Savaglio et al. 2005, Epinat et al. 2009, Förster Schreiber et al. 2009). For galaxies without observations of $\mathrm{H}_{\alpha}$ and $\mathrm{H}_{\beta}$ determining the extinction is harder and subject to assumptions which can influence the metallicity estimates. Metallicity estimates for $\mathrm{z}>3$ galaxies are sparse since the $\mathrm{H}_{\alpha}$ and for $\mathrm{z}>4$ galaxies the $\mathrm{H}_{\beta}$ lines are not observable from the ground and metallicities have to rely on line ratios involving $[\mathrm{OII}] \lambda 3727 \dot{\mathrm{A}}$ and $[\mathrm{OIII}] \lambda \lambda 4958 \dot{\mathrm{A}}$ and $5007 \dot{\mathrm{A}}$ (e.g. Maiolino et al. 2008) all of which can be significantly affected by extinction.

The $[\mathrm{OIII}] 88 \mu \mathrm{m} /[\mathrm{NII}] 122 \mu \mathrm{m}$ metallicity diagnostic ratio we presented above, can provide a suitable alternative for high-z galaxies, especially for luminous submm galaxies where line measurements in the rest-frame UV/optical regime are often challening. In what follows we derive metallicities for three high-z systems where suitable FIR FS lines exist either from Herschel or ALMA measurements.

\subsubsection{HLSW-01}

Using the FIR FS lines measured by Herschel in HLSW-01 (presented in Section 2) and the methodology outlined above 


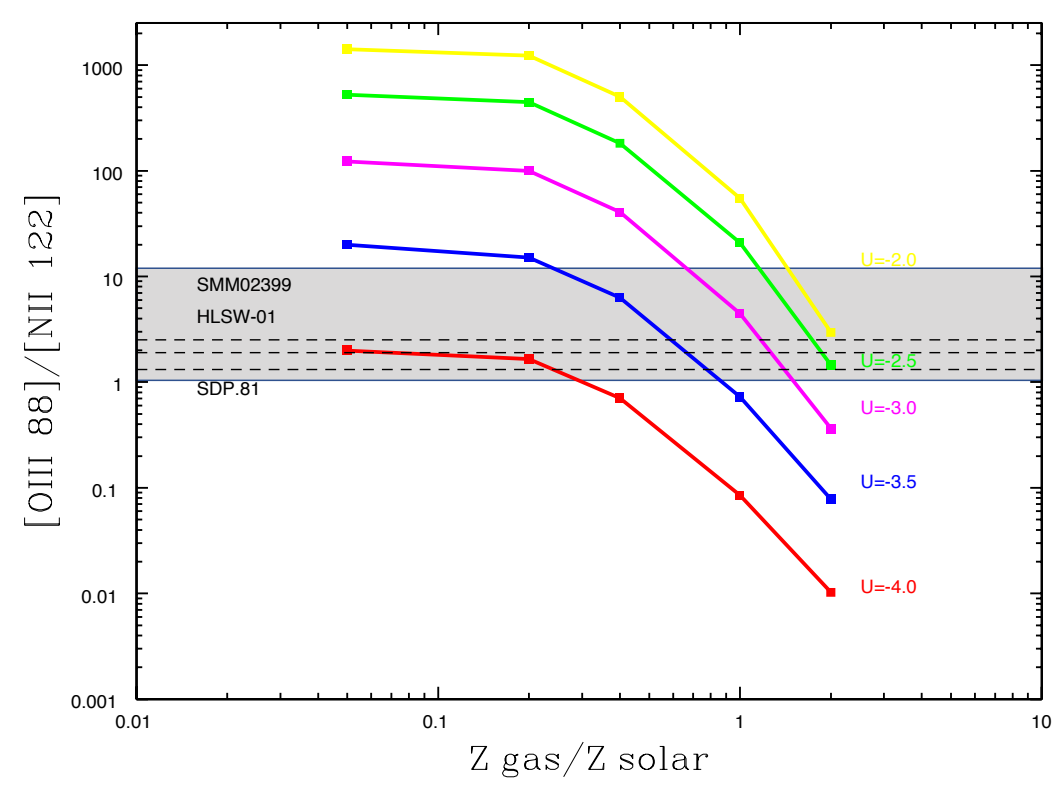

Figure 5. [OIII] $88 /[\mathrm{NII}] 122$ line ratio as a function of metallicity. The coloured lines (yellow, green, magenta, blue and red) correspond to different values of the ionization parameter $(\log \mathrm{U}=-2,-2.5,-3.0,-3.5,-4.0)$. For each curve we consider gas densities of log $\left(\mathrm{n} / \mathrm{cm}^{-3}\right): 1,2,3,4,5$. The dashed lines correspond to the [OIII] $88 /[\mathrm{NII}] 122$ values of SMM02399, HLSW-01 and SPD.81. The shaded region corresponds to the values of the Brauher et al. nearby galaxies.

we determine the gas phase metallicity for this system. As we discussed allready, the two most critical parameters of the models are the gas density and the ionization parameter. The [OIII] $52 /[\mathrm{OIII}] 88$ line ratio of 0.805 can be used to estimate the gas density: using Figure 1 (middle) from PS17 we estimate a gas density of $60-100 \mathrm{~cm}^{-3}$ corresponding to the [OIII] $52 /[\mathrm{OII}] 88$ line ratio measured for HLSW-01. The ionization parameter can be inferred from the $\mathrm{C}(88 / 122)$ continuum flux ratio. Using the SPIRE-FTS spectra we measure continuum fluxes of $318 \pm 10 \mathrm{mJy}$ and $210 \pm 11 \mathrm{mJy}$ around the $88 \mu \mathrm{m}$ and $122 \mu \mathrm{m}$ lines, respectively. We find a value of 1.51 for the $\mathrm{C}(88 / 122)$ ratio. Conley et al. (2011) and Wardlow et al. (2013) have reported SPIRE continuum measurements for HLSW-01. At the redshift of HLSW-01 of 2.975, $350 \mu \mathrm{m}$ corresponds to rest-frame $88.05 \mu \mathrm{m}$. Likewise, 500 $\mu \mathrm{m}$ corresponds to rest-frame $125.8 \mu \mathrm{m}$. Therefore, we can use the $350 \mu \mathrm{m} / 500 \mu \mathrm{m}$ ratio of the (de-boosted) fluxes measured by SPIRE as a proxy for $\mathrm{C}(88 / 122)$. We find that the ratio of the SPIRE fluxes is 1.49 , in good agreement with the $\mathrm{C}(88 / 122)$ continuum flux ratio we estimated from the SPIRE-FTS spectra. The $\mathrm{C}(88 / 122)$ ratio of 1.51 corresponds to a $\log (\mathrm{U})=-2.1$ (for an assumed gas density of $\left.100 \mathrm{~cm}^{-3}\right)$. Using the line fluxes reported in Table 1 we estimate a limit for the $[\mathrm{OIII}] 88 /[\mathrm{NII}] 122$ ratio of $>2.05$. This constrains the $\mathrm{Z}_{\text {gas }}$ to be $<0.8-1.0 \mathrm{Z}_{\odot}\left(\right.$ for $\left.\mathrm{n}_{\mathrm{H}}=100 \mathrm{~cm}^{-3}\right)$.

Since for HLSW-01 we detected the [OIII] $52 \& 88 \mu \mathrm{m}$ and $[\mathrm{NIII}] 57 \mu \mathrm{m}$ emission lines (reported in Table 1) we can use the formula $(2.2 \times[\mathrm{OIII}] 88 \mu \mathrm{m}+[\mathrm{OIII}]] 52 \mu \mathrm{m}) /[\mathrm{NIII}] 57 \mu \mathrm{m}$, to get an estimate of the metallicity. The two ratios should provide similar estimates of the metallicity since they are based on the same set of models. The advantage of using the ratio involving the three lines is that the scatter due to the dependency of the lines on the gas density is significantly reduced (see PS17 for a discussion). With a value of
5.44 for the $2.2 \times[\mathrm{OIII}] 88 \mu \mathrm{m}+[\mathrm{OIII}]] 52 \mu \mathrm{m}) /[\mathrm{NIII}] 57 \mu \mathrm{m}$ ratio, and assuming an ionization parameter $\log (\mathrm{U})=-2.1$ and gas density of $100 \mathrm{~cm}^{-3}$ we find that the gas phase metallicity for this object is $0.6<Z_{g a s} / Z_{\odot}<1.0$ in accordance with the limit derived from the $[\mathrm{OIII}[88 /[\mathrm{NII}] 122$ ratio. This value of the metallicity correspnds to $8.46<12+\log (O / H)<8.69$.

\subsubsection{SMJ02399}

The second galaxy for which we derive the metallicity is SMMJ02399, a luminous submm galaxy at $\mathrm{z}=2.803$ (Smail et al. 2002) lensed by the foreground cluster A370. Optical imaging and spectroscopy by Ivison et al. (1998) originally identified two components, a compact component (L1) hosting a narrow-line AGN (e.g. Villar-Martin et al. 1999), and a second, diffuse component (L2) associated with $\mathrm{L}_{\boldsymbol{\alpha}}$ and $\mathrm{H}_{\alpha}$ emission suggesting the presence of a strong starburst. Further analysis of NICMOS imaging (Aguirre et al. 2013) revealed a strong diffuse starburst component in L1.

Using the redshift ( $\mathrm{z}$ ) and Early Universe Spectrometer (ZEUS; Stacey et al. 2007), Ferkinhnoff et al. (2010) measured [OIII] $88 \mu \mathrm{m}$ line emission towards SMMJ02399. Based on the optical [OIII] $5007 \dot{\mathrm{A}} / \mathrm{H}_{\alpha}$ ratio, Ferkinhoff et al. (2010) argued that the $[\mathrm{OIII}] 88 \mu \mathrm{m}$ line emission is consistent with originating in $[\mathrm{HII}]$ regions. The $[\mathrm{NII}] 122 \mu \mathrm{m}$ line towards SMMJ02399 has been observed using both ZEUS (Ferkinhoff et al. 2011) and ALMA (Ferkinhoff et al. 2015). The [NII]122 $\mu \mathrm{m}$ line detection with ALMA is lower than the earlier detection with ZEUS and the authors attribute the discrepancy to the fact that the [NII] line flux is resolved out due to significantly more extended emission (and longer ALMA baselines) than expected. The spatial extent of the [NII] $122 \mu \mathrm{m}$ emission supports a starburst origin for the line. 
For the current estimates of the metallicity we use the [NII] $122 \mu \mathrm{m}$ line flux reported by Ferkinhoff et al. (2011) as we are interested in an integrated measurement of the [NII] $122 \mu \mathrm{m}$ line. We conclude that although SMMJ02399 contains an active nucleus (component L1) both the [OIII] $88 \mu \mathrm{m}$ and $[\mathrm{NII}] \mu \mathrm{m}$ line emission originate in the starburst component.

We measured the flux ratio $\mathrm{C}(88 / 122)$ using the continuum fluxes at 338 and $463 \mu \mathrm{m}$, which at the redshift of SMMJ02399 corresponds to rest frame $88 \mu \mathrm{m}$ and $122 \mu \mathrm{m}$, respectively. The value of the $\mathrm{C}(88 / 122)$ was found to be 1.4 which corresponds to values of the ionization parameter $-2.6<\log (\mathrm{U})<-2.1$ assuming gas densities between 10 and $1000 \mathrm{~cm}^{-3}$ (since for this object we cannot constrain the value of the gas density). The measured [OIII] $88 /[\mathrm{NII}] 122$ line flux ratio of 2.18 then corresponds to a metallicity of $0.7<\mathrm{Z}<1.1$ which translates to $8.53<\log (\mathrm{O} / \mathrm{H})<8.73$.

\subsubsection{SDP.81}

The third galaxy for which we derive an estimate of its metallicity is H-ATLAS J090311.6+003906 (SDP.81) at a redshift of of 3.042. Valtchanov et al. (2011) reported a $5 \sigma$ detection for the [OIII] $88 \mu \mathrm{m}$ line and and upper limit for the $[\mathrm{NIII}] 122 \mu \mathrm{m}$ line. Using the SPIRE 350 and $500 \mu \mathrm{m}$ continuum fluxes as a proxy for $\mathrm{C}(88 / 122)$ we find a value of 1.09 for the ratio. This value corresponds to a $-3.0<\log (\mathrm{U})<-$ 2.6 for values of gas density in the range 10 to $1000 \mathrm{~cm}^{-3}$, respectively. The lower limit for the $[\mathrm{OIII}] 88 /[\mathrm{NII}] 122$ line ratio is 1.45 . Using this line ratio together with the values of $\mathrm{U}$ and $\mathrm{n}$, in Figure 5, we determine an upper limit for the metallicity of SDP.81 of $\mathrm{Z}<2 \mathrm{Z}_{\odot}$ which corresponds to $\log (\mathrm{O} / \mathrm{H})<8.99$.

The finding that the gas phase metallicities of all three $\mathrm{z} \sim 3$ submm-luminous sources are consistent with solar values is in agreement with the normal CO line strengths found in them and other submm luminous galaxies and the apparent absence of an enhanced $\alpha_{\mathrm{CO}}$ conversion factor due to metallicity (e.g. Hodge et al. al. 2013, Aravena et al. 2016). In the next section, we shall use the FIR FS metallicity estimates to examine the location of the sources on the mass metallicity plane.

\section{MASS METALLICITY RELATION WITH FIR METALLICITY DIAGNOSTICS}

So far, studies of the mass-metallicity relation in nearby and distant galaxies have relied upon estimates of gas phase metallicities using strong line metallicity diagnostics from (rest-frame) optical emission lines. These line ratios have been calibrated against metallicities either 'directly' (through the use of the dependence of the metallicity on the electron temperature) or 'indirectly' (through photoionization models). However, it is well known that the output of these methods is often inconsistent with each other: metallicity estimates for the same galaxy can vary widely and introduce artificial evolutionary effects. Kewley and Ellison (2008) examined these trends and suggested conversion factors between different metallicity diagnostics. In this section we investigate the mass metallicity relation using new metallicity estimates derived from the FIR FS metallicity diagnostics.

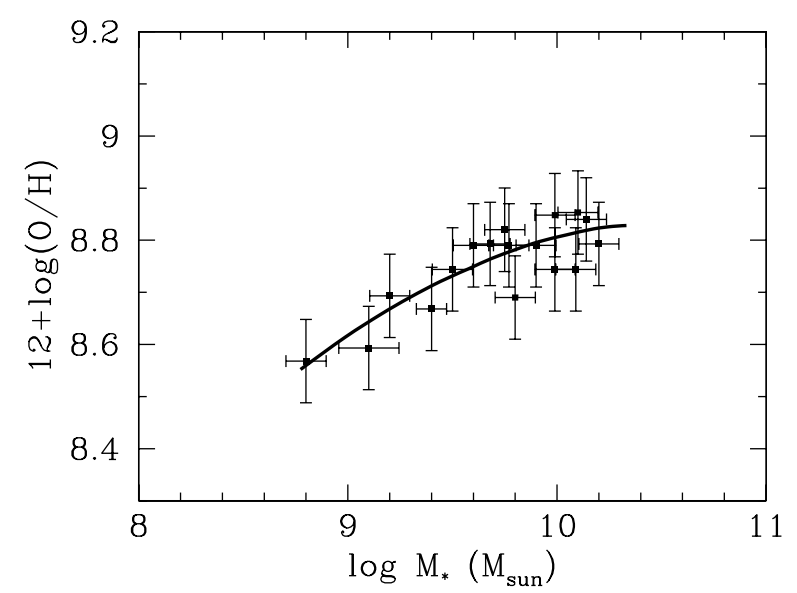

Figure 6. Mass-metallicity relation for the sample of nearby normal and starburst galaxies observed with ISO. The best fit mass-metallicity relation derived fro mthe local galaxies has the form $y=-0.098 x^{2}+2.0654 x-1.989$.

Figure 6 shows the mass-metallicity relation for the Brauher et al. (2008) sample of nearby galaxies. The stellar masses for the nearby galaxies have been taken from the literature or, using the mass-to-light ratio from Bell et al. (2003). The $(\mathrm{O} / \mathrm{H})$ abundance has been calculated from the metallicity values determined from our models using the value of $4.9 \times 10^{-4}$ for the solar O abundance (Asplund 2009) which corresponds to $12+\log (\mathrm{O} / \mathrm{H})=8.69 \pm 0.5$. Although the ISO sample of nearby galaxies contains only about two dozen galaxies, the plot in Figure 6 already shows some interesting trends: there seems to be a linear correlation between stellar mass and metallicity up to about $10^{9.5} \mathrm{M}_{\odot}$ after which the correlation appears to flatten. The flattening of the correlation at higher masses is also supported by the best fit to the data. A second order polynomial function provides a better fit $\left(\mathrm{R}^{2}=0.73\right)$ to the data in comparison to a linear (least square) fit $\left(\mathrm{R}^{2}=0.47\right)$. The second order polynomial fit to the data is of the form: $12+\log (\mathrm{O} / \mathrm{H})=-$ $0.098\left(\log \mathrm{M}_{*}\right)^{2}+2.065\left(\log \mathrm{M}_{*}\right)-1.989$ where $\mathrm{M}_{*}$ represents the stellar mass in units of solar masses. Although the ISO galaxies used in this study sample only a limited range in stellar mass, the fit to the data is in good agreement with e.g. the Tremonti et al. (2004) calibration both in the shape of the polynomial as well as the intercept to the y-axis.

Figure 7 shows the location of the three $\mathrm{z} \sim 3$ submmluminous galaxies for which we estimated their metallicity based on FIR FS diagnostic ratios on the $\mathrm{M}_{*}$-Z plot. Stellar masses for the three submm-luminous galaxies have been taken from the literature: Conley et al. (2011) derived a stellar mass of $\mathrm{M}_{*}=(6.3 \pm 3.4) \times 10^{10}\left(\mu_{L} / 10.9\right)^{-1} \mathrm{M}_{\odot}$ for HLSW01. Marques-Chaves et al. (2017, in prep) reanalysed the SED of HLSW-01 and derived a revised value for the stellar mass of $\mathrm{M}_{*}=1.0 \times 10^{11}\left(\mu_{L} / 10.9\right)^{-1} \mathrm{M}_{\odot}$. Aguirre et al. (2013) reported stellar masses for the three SMMJ02399 components: for the MZ plot we use the stellar mass calculated for the starburst component but the high end of the error bar on the stellar mass for this galaxy correspond to the total stellar mass of the system. Finally, for SDP.81 Negrello et al. 


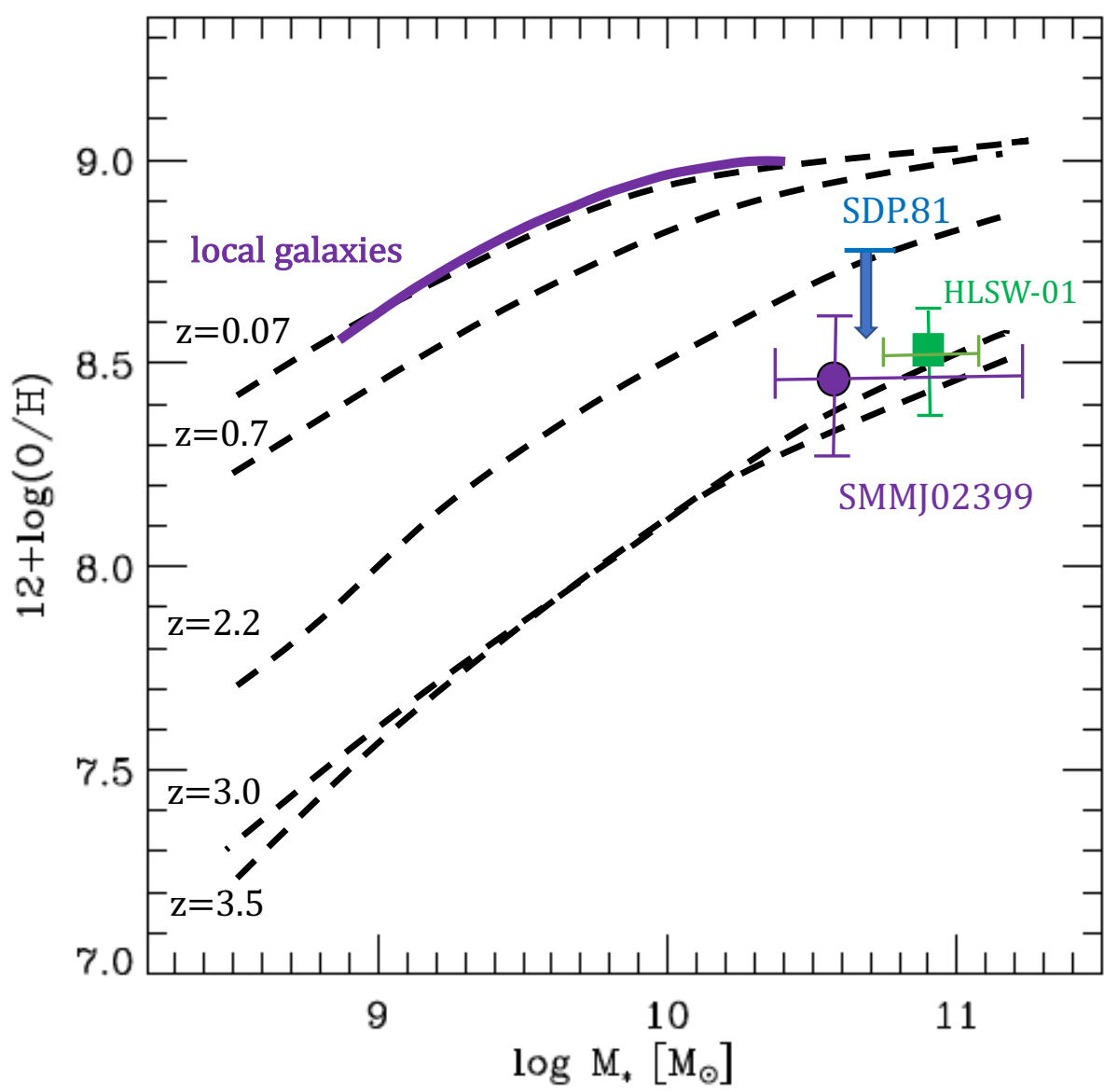

Figure 7. The mass-metallicity relation for the three high-z submm galaxies, HLSW-01, SMMJ03299 and SDP.81. The mass-metallicity relation derived in this work based on the local sample of ISO galaxies is shown in purple. The $\mathrm{z}=0.07$ relationship comes from Kewley \& Ellison (2008) while the $\mathrm{z}=0.7,2.2,3.0$ and 3.5 relationships come from Maiolino et al. 2008 and Mannucci et al. 2010. The coloured symbols represent the three submm-galaxies.

(2014) reported a stellar mass of $\mathrm{M}_{*}=6.6 \times 10^{10}\left(\mu_{L} / 10.6\right)^{-1}$ $\mathrm{M}_{\odot}$ where the magnification factor $\mu=10.6$ has been determined by Dye et al. (2014).

The vertical error bars show the uncertainty in $12+\log (\mathrm{O} / \mathrm{H})$ from the model predictions as well as the uncertainty in the measured $[\mathrm{OIII}] 88 /[\mathrm{NII}] 122$ line ratio. The horizontal bar shows the uncertainty in stellar mass (or in the case of SMMJ03299 the range of stellar mass estimates). The dashed lines in Figure 7 show the mass-metallicity relations at different redshifts taken from the literature, the $\mathrm{z}=0.07$ has been taken from Kewley \& Ellison (2008), $\mathrm{z}=0.7$, 2.2 and 3.5 from Maiolino et al. (2008) and $z=3.0$ from Mannucci et al. (2009). We also show the local mass-metallicity relationship we derived in Section 4 . The metal content of the three $\mathrm{z} \sim 3$ submm-luminous galaxies to be in accordance with that expected from the mass-metallicity relation for that particular mass.

We have shown that FIR FS line ratios provide consistent metallicity estimates for galaxies near and far. Detection of [OIII] 88 and [NII] $122 \mu \mathrm{m}$ emission lines is now possible with ALMA for galaxies at $z>4$ where optical lines might be out of reach from the ground.

\section{CONCLUSIONS}

Photoionization models for the FIR FS lines found in the spectra of star-forming galaxies were presented in PS17. Combinations of various FIR FS line ratios were examined to find those that can be used to determine gas phase metallicities in galaxies. Here, we focused on the applicability of the $[\mathrm{OIII}] 88 / \mathrm{NII}] 122$ line ratio as a potential metallicity diagnostic. This diagnostic ratio depends strongly on the value of the ionization parameter and only mildly on the value of the gas density. We showed that the $\mathrm{C}(88 / 122)$ ratio correlates well with the $\mathrm{C}(60 / 100)$ which is sensitive to the ionization parameter and the shape of the underlying SED. Hence, we suggest that the $\mathrm{C}(88 / 122)$ ratio can be used to constrain the value of the ionization parameter while the addition of the $[\mathrm{NII}] 205 \mu \mathrm{m}$ emission line can help constrain the gas density. We applied our methodology to a sample of local normal and star-forming galaxies and distant $\mathrm{z} \sim 3$ submm-luminous galaxies. The results of our work can be summarised as follows:

(i) The $[\mathrm{OIII}] 88 \mu \mathrm{m} /[\mathrm{NII}] 122 \mu \mathrm{m}$ emission line ratio provides a good estimate of the gas phase metallicities in a sample 
of local normal and star-forming galaxies with ISO FIR FS line measurements. The continuum flux ratio $\mathrm{C}(88 / 122)$ was used to determine the ionization parameter $\mathrm{U}$ while the [NII] $122 \mu \mathrm{m} / 205 \mu \mathrm{m}$ line ratio was used to estimate gas densities. We constructed the mass-metallicity relationship and found that its shape and y-intercept is similar to that derived by e.g. Tremonti et al. (2004) based on optical emission lines. (ii) We presented new, previously unpublished, SPIRE-FTS detections of a number of FIR FS lines from the strongly lensed submm-luminous galaxy HLSW-01 at $\mathrm{z}=2.9758$. [OIII] 52 and $88 \mu \mathrm{m},[\mathrm{NIII}] 57 \mu \mathrm{m},[\mathrm{OI}] 63 \mu \mathrm{m}$ and [CII] 158 $\mu \mathrm{m}$ were detected at $>3 \sigma$ while $3 \sigma$ limits were derived for [OI]145 $\mu \mathrm{m}$ and [NII] $122 \mu \mathrm{m}$. We used the [OIII] $88 /[\mathrm{NII}] 122$ line ratio and found that the gas phase metallicity of the system is close to solar. The solar value for the metallicity of this system was also found using a second diagnostic ratio combining [OIII] $88,[\mathrm{OIII}] 52$ and $[\mathrm{NIII}] 57 \mu \mathrm{m}$.

(iii) The $[\mathrm{OIII}] 88 /[\mathrm{NII}] 122$ line ratio was used to estimate gas phase metallicities for another two $\mathrm{z} \sim 3$ submm luminous galaxies SMMJ0399 and SDP.81 using published data. The metallicities for these systems are consistent with solar values. We found that all three $\mathrm{z} \sim 3$ submm-luminous galaxies studied here exhibit lower metallicities, for a fixed stellar mass $\mathrm{M}_{*}$, compared to the calibration defined for local $(\mathrm{z}=0)$ galaxies. However, the mass-metallicity relation of the three submm-luminous galaxies agrees well with the one defined by other samples at the same redshift.

The [OIII] $88 /[\mathrm{NII}] 122$ line ratio can be a potentially powerful diagnostic of gas phase metallicities for $\mathrm{z}>4$ galaxies where most optical emission lines, traditionally used to establish metallicities, shift outside of reach from the ground. ALMA can observe these FIR FS lines from high redshift systems opening the way for establishing metallicities in the very early Universe.

\section{ACKNOWLEDGEMENTS}

We thank the referee, Graziano Ucci, for his useful comments and suggestions. D.R. and M.P-S acknowledge support from STFC through grant ST/N000919/1. MPS acknowledges support from the John Fell Oxford University Press (OUP) Research Fund and the University of Oxford. IPF acknowledges support from the Spanish Ministerio de Economia y Competitividad (MINECO) under grant number ESP2015-65597-C4-4-R. D.Rie. acknowledges support from the National Science Foundation under grant number AST-1614213 to Cornell University. SPIRE has been developed by a consortium of institutes led by Cardiff University (UK) and including Univ. Lethbridge (Canada); NAOC (China); CEA, LAM (France); IFSI, Univ. Padua (Italy); IAC (Spain); Stockholm Observatory (Sweden); Imperial College London, RAL, UCL-MSSL, UKATC, Univ. Sussex (UK); and Caltech, JPL, NHSC, Univ. Colorado (USA). This development has been supported by national funding agencies: CSA (Canada); NAOC (China); CEA, CNES, CNRS (France); ASI (Italy); MCINN (Spain); SNSB (Sweden); STFC, UKSA (UK); and NASA (USA). This research has made use of the NASA/IPAC Extragalactic Database (NED) which is operated by the Jet Propulsion Laboratory, California Institute of Technology, under contract with the National Aeronautics and Space Administration.

\section{REFERENCES}

Abel, N.P, Ferland, G. J., Shaw, G., van Hoof, P. A. M. et al., 2005, ApJS, 161, 65

Abel, N.P., Dudley, C., Fischer, J., Satyapal, S., van Hoof, P. A. M. et al., 2009, ApJ, 701, 1147

Aguirre, P., Baker, A. J., Menanteau, F., Lutz, D., Tacconi, L. J. 2013, ApJ 768, 164

Asplund, M., Grevesse, N., Sauval, A. J., Scott, P., 2009, ARA\&A, 47,481

Brauher, J.R., Dale, D., A., Helou, G., 2008, ApJS, 178, 280

Chanial, P., et al., 2007, A\&A 462, 81

Conley, A., et al., 2011, ApJ Lett, 732, 35

Dekel., A., Sari, R., Ceverino, D., 2009, ApJ 703, 785

Davé, R., Finlator, K., Oppenheimer, B.D., 2012, MNRAS, 421, 98

Gehrz, R.D., Beclin, E.E., de Pater, I., et al., 2009, Advances in Space Research, 44, 413

Keres, D., Katz, N., Fardal, M., Dave, R.,\& Weinberg, D. H. 2009, MNRAS, 395, 160

Genzel, R., Tacconi, L. J., Gracia-Carpio, J., et al. 2010, MNRAS, 407, 2091

Conroy, C., \& Wechsler, R.H., 2009, ApJ, 696, 620

Dopita, M., et al., 2006, ApJ 647, 244

Dye, S., Negrello, M., Hopwood, R., et al., 2014, MNRAS, 440, 2013

Eales, S., et al. 2010, PASP, 122, 499

Erb, D., Shapley, A. E., Pettini, M., et al., 2006, ApJ, 644, 813

Faisst, A.L., Capak, P.L., Davidzon, I., et al., 2016, ApJ, 822, 29

Ferkinhoff, C., Hailey-Dunsheath, S., Nikola, T., et al., 2010, ApJ Lett, 714, 147

Ferkinhoff, C., Brisbin, D., Nikola, T., et al., 2011, ApJ, 740, 29

Ferkinhoff, C., Brisbin, D., Nikola, T., et al., 2015, ApJ, 806, 260

Ferland, G., et al., 2013, MxAA, 49, 49, 137

Fischer, J., Abel, N.P., Gonzalez-Alfonso, E., et al., 2014, ApJ, 795,117

Förster Schreiber, N. M., Genzel, R., Bouché, N., et al., 2009, ApJ 706, 1364

Gavazzi, R., Cooray, A., Conley, A., et al. 2011, ApJ738, 125

Griffin, M.J., Aberghel, A., Abreu, A., et al., 2010, A\&A, 518, 3

Herrera-Camus, R., Bolatto, A., Smith, J.D., et al., 2016, ApJ, 826,175

Hodge, J.A., Carilli, C.L., Walter, F., Daddi, E., Riechers, D.,2013, ApJ, 776,22

Kessler, M.F., Steinz, J.A., Anderegg, M.E., et al., 1996, A\&A, 315,27

Kewley, L., Dopita, M.A., 2002, ApJS, 142, 35

Kewley, L., Ellison, S., 2008, ApJ, 681, 1183

Kilerci Eser, E., Goto, T., Doi, Y., 2014, ApJ 797, 54

Leitherer, C., Schaerer, D., Goldader, J., et al., 1999, ApJS, 123, 3

Magdis, G.E., Rigopoulou, D., Hopwood, R., et al., 2014, ApJ, 796,63

Maiolino, R., et al., 2008, A\&A, 488, 463

Mannucci, F., Cresci, G., Maiolino, R., Marconi, A., Gnerucci, A. et al., 2010, MNRAS, 408, 2115

Moustakas, J., Kennicutt, R.C., 2006, ApJ, 651, 155

Nagao, T., Maiolino, R., Marconi, A., Matsuhara, H., 2011, A\&A, 526,149

Negrello, M., Hopwood, R., Dye, S., et al., 2014, MNRAS, 440, 1999

Oberst, T.E., Parshley, S.C., Stacey, G.J., et al., 2006, ApJ, 652, 125

Oliver, S. J., Bock, J., Altieri, B., et al. 2012, MNRAS, 424, 1614

Onodera, M., Carollo, C. M., Lilly, S., et al. 2016, ApJ, 822, 1

Pagel, B. E. J., Edmunds, M. G., Blackwell, D. E., Chun, M. S., Smith, G. 1979, MNRAS, 189, 95

Pereira Santaella, M., Rigopoulou, D., Farrah, D., Lebouteiller, 
V., Li, J., 2017, MNRAS, 470, 1218

Pilbratt, G., G. L.; Riedinger, J. R., Passvogel, T., et al., 2010, A\&A, 518,1

Riechers, D., Cooray, A., Neri, R., et al., 2011, ApJ Lett. 733, 12

Rigopoulou, D., Hurley, P., Swinyard, B.M., et al., 2013, MNRAS, 434,2051

Rigopoulou, D., Hopwood, R., Magdis, G.E., et al., 2014, ApJ Lett., 718,15

Rupke, D., S.N., Veilleux, S., Baker, A. J., 2008, ApJ, 674, 172

Savaglio, S., Glazebrook, K., Abraham, R. G., et al. 2004, ApJ, 602,51

Scott, K. S., Lupu, R. E., Aguirre, J. E., et al., 2011, ApJ, 733, 29

Smail, I., Owen, F. N., Morrison, G. E., et al., 2002, ApJ 581, 844

Sommariva, V., Mannucci, F., Cresci, G., et al. 2012, A\&A, 539, 136

Storchi-Bergman, T., Calzetti, D., Kinney, A. L., 1994, ApJ 429, 572

Swinyard, B.M., Polehampton, E. T., Hopwood, R., et al. 2014, MNRAS, 440, 3658

Thatte, N., Tecza, M., Clarke, F., et al., 2006, SPIE, 6269, 3

Tremonti, C.A, Heckman, T.M., Kauffmann, G., et al. 2004, ApJ, 613,898

Valtchanov, I., Virdee, J., Ivison, R.J., et al., 2011, MNRAS, 415,3473

van Hoof, P. A. M., Weingartner, J. C., Martin, P. G., Volk, K., Ferland, G. J., 2004, MNRAS, 350, 1330

Wuyts, E., Kurk, J., Foerster, Schrieber, N.M., et al., 2014, ApJ 789,40

This paper has been typeset from a $\mathrm{T}_{\mathrm{E}} \mathrm{X} / \mathrm{LA} \mathrm{T} \mathrm{E}$ file prepared by the author. 